\title{
Ethnicity Moderates the Outcomes of Self-Enhancement and Self-Improvement Themes in Expressive Writing
}

\author{
William Tsai, Anna S. Lau, Andrea N. Niles, Jordan Coello, Matthew D. Lieberman, Ahra C. Ko, \\ Christopher Hur, and Annette L. Stanton \\ University of California, Los Angeles
}

\begin{abstract}
The current study examined whether writing content related to self-enhancing (viz., downward social comparison and situational attributions) and self-improving (viz., upward social comparison and persistence) motivations were differentially related to expressive writing outcomes among 17 Asian American and 17 European American participants. Content analysis of the essays revealed no significant cultural group differences in the likelihood of engaging in self-enhancing versus self-improving reflections on negative personal experiences. However, cultural group differences were apparent in the relation between self-motivation processes and changes in anxiety and depressive symptoms at 3-month follow-up. Among European Americans, writing that reflected downward social comparison predicted positive outcomes, whereas persistence writing themes were related to poorer outcomes. For Asian Americans, writing about persistence was related to positive outcomes, whereas downward social comparison and situational attributions predicted poorer outcomes. Findings provide evidence suggesting culturally distinct mechanisms for the effects of expressive disclosure.
\end{abstract}

Keywords: expressive writing, culture, self-enhancement, self-improvement, Asian

The positive effects of expressive writing (EW) on psychological and physical health are well documented (see Frattaroli, 2006, for a review). However, there is still much to be learned about the mechanisms underlying the ameliorative effects of EW, and the wide variability in effect sizes across studies suggests possible moderators of efficacy. Recent findings suggest cultural differences in the benefits of EW, but these studies raise more questions than answers because studies have variably revealed that individuals of Asian descent benefited more (Lu \& Stanton, 2010) and less (Knowles, Wearing, \& Campos, 2011) than European Americans. Examination of processes undertaken by individuals during EW may help to explain differential outcomes across cultural contexts. In this study, we examined whether the approach to writing about stressors would enhance outcomes to the extent that it is in line with broader cultural goals. Using data from a trial of EW (Niles, Haltom, Mulvenna, Lieberman, \& Stanton, in press), we examined whether self-relevant motivations (i.e., writing content related to self-enhancing and self-improving motivations) would predict EW outcomes distinctly across cultural groups.

This article was published Online First August 11, 2014.

William Tsai, Anna S. Lau, Andrea N. Niles, Jordan Coello, Matthew D. Lieberman, Ahra C. Ko, Christopher Hur, and Annette L. Stanton, Department of Psychology, University of California, Los Angeles.

This work was supported by the National Institutes of Health under Grant R01MH084116 awarded to Matthew D. Lieberman and Annette L. Stanton. We declare no potential conflicts of interests with respect to the authorship and/or publication of this article.

Correspondence concerning this article should be addressed to Annette L. Stanton, University of California, Los Angeles, 1285 Franz Hall, Box 951563, Los Angeles, CA 90095. E-mail: astanton@ucla.edu

\section{Cultural Differences in Self-Enhancement and Self-Improvement}

Self-enhancement involves maintaining positive self-regard by focusing and elaborating on positive information about the self (Heine \& Hamamura, 2007; Taylor \& Brown, 1988). Crosscultural research demonstrates that self-enhancing motivations appear weaker among individuals of East Asian descent compared with European Americans (Heine \& Hamamura, 2007), a pattern related to East-West differences in self-construal (Markus \& Kitayama, 2010). East Asian cultures foster a view of the self as interdependent and defined by relational context, in which behavior is dictated by the social situation, so that the self is malleable and responsive to social context and referenced against social standards rather than being fixed. Thus, interdependent individuals are accustomed to holding a self-critical stance and learning to exercise vigilance to areas in which one is falling short of group standards in service of social harmony. In contrast, European Americans tend to view the self as an independent entity defined by internal fixed attributes that remain consistent across contexts (Markus \& Kitayama, 1991). Under the entity view, when confronted with a threat to self, restorative self-enhancement becomes crucial (Heine \& Hamamura, 2007). In contrast, within an incremental view, self-improvement is propelled by open consideration of one's shortcomings and an emphasis on struggling with difficulty (Heine, Lehman, Markus, \& Kitayama, 1999; Stevenson, 1994).

Whereas European Americans achieve positive self-regard through self-enhancement, East Asians achieve positive selfregard through self-improvement and maintaining one's standing with others (Heine \& Hamamura, 2007). Supporting these theories, European Americans who maintain positive self-views through self-enhancement report having greater psychological well-being 
(Taylor \& Brown, 1988). On the other hand, East Asians report having greater psychological well-being and higher self-worth when they focus on maintaining or improving their interpersonal relationships (Kang, Shaver, Sue, Min, \& Jing, 2003). These findings support the hypotheses that individuals who reflect on stress experiences in a culturally syntonic manner (i.e., Westerners engaging in self-enhancement and Easterners engaging in selfimprovement) may report better psychological well-being. However, what remains unclear is whether individuals who reflect on stressors in a culturally dystonic manner (i.e., Westerners engaging in self-improvement and Easterners engaging in self-enhancement) will report greater distress. In the present study, we addressed this question by examining how writing content related to self-relevant motivations predicts EW outcomes distinctly across cultural groups.

\section{Processes Supporting Self-Enhancement: Self-Serving Attributions and Downward Social Comparison}

When reflecting on stressful personal experiences, situational attributions can enhance positive self-views. When "blame" is laid elsewhere, one can preserve the view of the self as efficacious (Bandura, 1999). There is some evidence that situational attributions for failure are more common among European Americans than East Asians. European Americans are more likely to characterize assessments as not credible when they experience failure, whereas Japanese individuals are less likely to endorse the credibility of tests when they succeed (Heine et al., 2001). Indeed, Westerners are significantly more likely than East Asians to make dispositional attributions for positive events and situational attributions for negative events (Mezulis, Abrahmson, Hyde, \& Hankin, 2004).

Another strategy that can deflect threat is social comparison. Individuals may seek social comparison information in ways that promote self-enhancement or self-improvement goals. Social comparison can involve comparing oneself with others doing better (i.e., upward social comparison) or worse (i.e., downward social comparison) than the self. Downward social comparison is a self-enhancing strategy for restoring positive self-regard when self-esteem is threatened (Crocker, Thompson, McGraw, \& Ingerman, 1987; Wills, 1981). In Western samples, individuals who attend to their strengths through downward social comparison report greater life satisfaction and less depression (Diener, 1984; Taylor \& Brown, 1988). However, cross-cultural studies suggest that European Canadians are more likely than East Asians to engage in downward social comparisons in their spontaneous self-descriptions (Ross, Heine, Wilson, \& Sugimori, 2005) and when reflecting on negative life events (White \& Lehman, 2005a) in ways driven by self-enhancement motives.

\section{Processes Supporting Self-Improvement: Upward Social Comparison and Persistence Facing Failure}

Complementing the findings above, East Asians appear more likely than Westerners to engage in upward social comparison, consistent with a self-improving or mastery orientation focus (Butler, 1992). When given performance feedback and an opportunity to compare themselves with better or worse performers, Asian Canadians were more likely than European Canadians to seek upward social comparisons (White \& Lehman, 2005b). In the context of academic self-assessments, students with greater interdependent values reported a greater desire to make upward comparisons and lower desire to make downward comparisons compared with those low in interdependent values (Chung \& Mallery, 1999).

A second process that supports self-improving motives involves pursuing challenges that are tied to past failure. Easterners tend to subscribe to incremental theories of ability that suggest that achievement hinges on effort not talent, making persistence crucial (Heine \& Hamamura, 2007). Indeed, whereas Japanese participants are more likely to continue working on a difficult task if they have been given failure feedback as opposed to success feedback, European Americans are more likely to elect a novel task not associated with past failure (Heine et al., 2001). Japanese individuals judge failures as having more influence on self-esteem than successes, whereas the converse applies to North Americans (Kitayama, Markus, Matsumoto, \& Norasakkunkit, 1997).

\section{The Current Study}

In the current study, we conducted content analysis of essays from Niles et al. (in press) to examine cultural differences in the use and impact of attributions, social comparison, and persistence themes in EW. Niles and colleagues found no significant main effects of writing condition on anxiety, depressive, or physical symptoms in a sample of 116 participants $\left(M_{\text {age }}=21.2\right.$ years, $S D=2.89$ ); however, trait emotional expressiveness moderated the effects of EW. The goal in the present analysis was to elucidate potential cultural variability in psychological mechanisms underlying the therapeutic benefits of EW. We predicted that Asian Americans would engage in more self-improvement writing, whereas European Americans would engage in more selfenhancement writing. Moreover, we predicted that the socialcognitive processes apparent in participants' writing would have positive implications for psychological and physical functioning when it is syntonic with the writer's culturally modal self-motives. That is, we hypothesized that cultural group would moderate the effects of social comparison, attributions, and persistence focus, with Asian Americans benefiting more from self-improving themes in their writing and European Americans benefiting more from writing reflecting self-enhancement motives.

\section{Method}

\section{Participants}

Participants included 34 Asian American and European American adults who participated in a randomized study of 116 adults on EW. Eligibility criteria were ages between 18 and 40 years, fluency in English, no self-reported psychiatric disorder or serious physical illness, and having experienced a stressful event within the past 5 years that they rated as 5 or greater in stressfulness on a 7-point Likert scale. A total of 116 participants met eligibility and were randomly assigned to the EW $(n=59)$ and control $(n=$ $57)$ conditions. The current analysis focused only on individuals in the EW condition who self-identified as Asian American $(n=17)$ or European American $(n=17)$. All 34 participants used in the present study completed the follow-up questionnaire. Participants 
were $19(55 \%)$ men and were an average of 20.76 years old $(S D=$ 3.31, range $=18-35$ years). Among the 17 Asian Americans, 12 (71\%) were born in the United States, and five were foreign-born. The Asian American participants included eight Chinese (47\%), one Korean (6\%), two Japanese (12\%), three Filipino (18\%), two Vietnamese (12\%), and one participant who declined to state. Among the 17 European Americans, 14 (82\%) were born in the United States, and three were foreign-born.

\section{Procedure}

Participants were students and adults recruited from introductory psychology courses and fliers posted in the community. In a baseline session (Time [T] 1), they provided consent, completed questionnaires, and completed a functional MRI scan. Participants then engaged in four 20-min writing sessions, scheduled at least 3 days apart and occurring within the 8 weeks following T1. Participants completed the four writing sessions in an average of 24.83 days $(S D=$ 5.16). During the initial writing session, participants were assigned randomly to a writing condition (EW or control). In the EW condition, participants wrote about their "deepest thoughts and feelings" regarding their "most stressful or traumatic experience during the past 5 years." Three months after the final writing session, participants completed follow-up questionnaires online (T2).

\section{Dependent Measures}

Depressive symptoms. A composite scale was created that comprised three measures of depressive symptoms (Niles et al., in press): the seven-item Depression subscale of the Depression, Anxiety, and Stress Scale (DASS-21; e.g., "I couldn't seem to experience any positive feeling at all"; Antony, Bieling, Cox, Enns, \& Swinson, 1998), the Beck Depression Inventory 1A (BDI-1A; e.g., "I feel I am a complete failure as a person"; Beck \& Steer, 1984), and the 20-item Center for Epidemiologic Studies Depression Scale (CES-D; e.g., "I felt depressed"; Radloff, 1977). All are psychometrically sound measures of depressive symptoms (Antony et al., 1998; Radloff, 1977; Steer, Beck, Garrison, \& Lester, 1988). Standardized scores on the three measures were averaged at $\mathrm{T} 1$ and $\mathrm{T} 2$ to create composite scores, which had a mean of 0 and standard deviation of 1 at T1. Correlations between depression measures at $\mathrm{T} 1$ ranged from .63 to .78 , and at T2, from .60 to .86. In this sample, alphas were $.86, .80, .62$ for Asian Americans and .81, .74, .70 for European Americans at T1, and $.88, .94, .85$ for Asian Americans and $.84, .78, .91$ for European Americans at T2 for the DASS-21, BDI-1A, and CES-D, respectively.

Anxiety symptoms. A composite measure was created from standardized scores on three measures of anxiety symptoms: the seven-item Anxiety subscale of the DASS-21 (e.g., "I found myself in situations that made me so anxious I was most relieved when they ended.") and the Anxiety and Somatization subscales from the Brief Symptom Inventory (BSI; e.g., "Suddenly scared for no reason" and "Faintness or dizziness"; Derogatis \& Melisaratos, 1983). Correlations between anxiety scales at T1 ranged from .61 to .63 and at $\mathrm{T} 2$ from .81 to .83 . In this sample, alphas for the Anxiety subscale were $.90, .87, .89$ for Asian Americans and .67 , .74, .60 for European Americans at T1, and .83, .90, and .70 for Asian Americans and .81, .91, .83 for European Americans at T2 for the BSI-Anxiety, BSI-Somatization, and DASS-21-Anxiety subscales, respectively.
Physical symptoms. The 54-item Pennebaker Inventory of Limbic Languidness (Pennebaker, 1982) assesses a number of common physical symptoms (e.g., "chills," "tightness in chest," "nausea"). Participants indicate how often they have experienced each symptom on a 5-point Likert scale $(1=$ never or almost never, $5=$ more than once every week). In this sample, alphas were .91 for Asian Americans and .96 for European Americans at T1 and .95 for Asian Americans and .93 for European Americans at T2.

\section{Essay Coding}

The 134 essays (33 participants with four essays, one participant with two essays) from the EW condition were content analyzed by coders who were unaware of the study hypotheses and participants' demographic information. The first author trained three Asian Americans and one Latino American coder to use a manual adapted from Niles, Haltom, Hur, Lieberman, and Stanton (2014) and Creswell et al. (2007). Coders were trained to detect the presence of a situational attribution for the negative event, social comparison, and persistence themes in essays, with a sentence being the smallest unit of text that could be coded. Coders went through the essays line-by-line and codes were applied to "meaning units," which could span a single sentence or multiple consecutive sentences. A form of dichotomous interval coding was used, such that codes were either present or absent within a five-line block of text (e.g., Lines 1-5, 6-10, and 11-15). Thus, although multiple meaning units might be found within a five-line block, only a unit's presence was recorded. If a single meaning unit spanned multiple line blocks, the attached code was counted in multiple blocks (e.g., a meaning unit spanning Lines $4-8$ would be noted in two line blocks). We used dichotomous interval coding and not summed frequency counts of sentences to be consistent with how we calculated interrater reliability. Codes were not considered mutually exclusive. Thus, meaning units could contain multiple codes. A limitation of this approach is that overlapping coding may lead to violating the statistical assumption of independence of observations when multiply coded variables are entered into a statistical model. Thus, each category of essay codes was assessed alone.

Coders were trained using 12 randomly selected training essays, and then all four independently coded an additional 28 essays (19.2\% of the sample). Interrater reliability was assessed based on the posttraining essays. Agreement was satisfactory across the codes for situational attribution (85-90\% agreement; kappa between pairs of coders ranged from 0.70 to 0.82 ), downward social comparison (92.5-97.5\% agreement; kappa ranged from 0.60 to 0.82), upward social comparison (90-95\% agreement; kappa ranged from 0.65 to 0.85 ), and persistence (90-95\% agreement; kappa ranged from 0.70 to 0.87 ). As such, the remaining essays were content analyzed by single coders.

Formal operational definitions and text examples of each coding category are provided in Table 1 . We defined downward social comparison as "comparison with others doing worse than the self" and upward social comparison as "comparison with others doing better than the self." The self needed to be explicitly mentioned in a comparison with others to be coded. Situational attribution was defined as "explaining negative personal events with external attributions," such that codes were applied when themes of "blaming others or the context for personal failures" appeared. Persis- 
Table 1

Definitions and Examples of Self-Enhancement and Self-Improvement Codes by Ethnicity

\begin{tabular}{|c|c|c|}
\hline Code & European American & Asian American \\
\hline \multicolumn{3}{|l|}{ Self-enhancement } \\
\hline $\begin{array}{l}\text { Situational attributions for negative events: } \\
\text { Explaining negative personal events with } \\
\text { external attributions }\end{array}$ & $\begin{array}{l}\text { "I don't know why it is but I don't do very } \\
\text { well in a stable environment." }\end{array}$ & $\begin{array}{l}\text { "Stress from school always seemed to affect the } \\
\text { relationship, not enough time for each other, } \\
\text { worrying about poor grades." }\end{array}$ \\
\hline $\begin{array}{l}\text { Downward social comparison: Comparison with } \\
\text { others doing worse than the self }\end{array}$ & "My other siblings easily drop the ball." & $\begin{array}{l}\text { "I can handle the stress of certain tests that } \\
\text { people deemed too difficult." }\end{array}$ \\
\hline \multicolumn{3}{|l|}{ Self-improvement } \\
\hline $\begin{array}{l}\text { Upward social comparison: Comparison with } \\
\text { others doing better than the self }\end{array}$ & $\begin{array}{l}\text { "I can't help but feel uneasy and useless, } \\
\text { not good enough when my friends seem } \\
\text { to be getting so far ahead, yet I feel like } \\
\text { I've back-tracked } 20 \text { steps." }\end{array}$ & $\begin{array}{l}\text { "Looking at great men who came before me, as } \\
\text { role models, I should strive to emulate their } \\
\text { consistency and composure." }\end{array}$ \\
\hline $\begin{array}{l}\text { Persistence: Actively thinking about persevering } \\
\text { through conflicts/stressors }\end{array}$ & $\begin{array}{l}\text { "It is amazing how I was still able to do } \\
\text { well in high school and come here to } \\
\text { university." }\end{array}$ & $\begin{array}{l}\text { "I was proud of my tenacity-I pulled through } \\
\text { that experience and now I am a better person } \\
\text { because of it." }\end{array}$ \\
\hline
\end{tabular}

tence was defined as "the process of actively thinking about persevering and working through adversity/stress/failure." As such, codes were applied when themes of "sticking with it" and "not giving up" appeared. Statements simply describing negative feelings, thoughts, and events did not qualify as persistence. Persistence had to involve writing about the negative events/experiences in a way that demonstrated drive for overcoming failure.

\section{Covariates: Objective Threat, Number of Positive/Negative Emotion Words, and Word Count}

We also controlled for age and gender because previous research has shown that these variables can influence the effectiveness of EW (Smyth, 1998). In addition, we included covariates related to the stressor reflected on in the EW and other characteristics of EW that may impact intervention outcomes.

Because the nature of the traumas written about might have been a moderating variable (Smyth, 1998), we examined the objective threat associated with the stressor as a covariate. Procedures for rating the severity of stressful life events outlined in the UCLA Life Stress Interview protocol were used (Hammen, Marks, Mayol, \& DeMayo, 1985). Two coders rated the stressfulness of the event on a 5 -point Likert scale $(1=$ minimal impact, $5=$ worst impact $)$ and then discussed their impressions to come to a consensus. Severity ratings were based on the number of life domains affected by the event (e.g., social, work/school) as well as how severely the area was affected. Prior to consensus rating, the two coders' independent ratings were highly correlated $(r=.74)$.

We controlled for the number of positive and negative emotion words in the EW essays because emotional expressivity can predict health outcomes of EW (e.g., Lepore \& Greenberg, 2002; Pennebaker, Mayne, \& Francis, 1997). Our goal was to determine whether self-relevant motivations predicted outcomes independently of negative or positive affectivity during EW. The number of positive and negative emotion words was identified using the Linguistic Inquiry and Word Count program (Francis \& Pennebaker, 1993), which calculates the percentage of total words written that were either positively emotionally valenced (e.g., love, nice, sweet) or negatively valenced (e.g., hurt, ugly).

Word count was included as a covariate to control for overall engagement during EW and to address the potential for essay length to be confounded with the frequencies of each code of interest. Longer essays are more likely to yield higher code frequency than shorter essays.

\section{Statistical Analyses}

Data were examined for outliers ( $>3 S D$ from the mean) on outcomes and for EW codes. Three outliers were identified for anxiety and one outlier was identified for depressive symptoms. Outliers were replaced with the next highest value based on the Winsor method (Guttman, 1973). Testing the normality of the predicted residuals for $\mathrm{T} 2$ anxiety symptoms revealed a slight positive skew. Thus, we also tested our anxiety models using Poisson regression; the results remained the same as using linear regression. We report linear regression results because no comparable approximations of $R^{2}$ effect sizes currently exist for Poisson regression.

To examine cultural differences in the associations between essay codes and psychological and physical well-being at T2, we conducted a series of linear regression analyses separately for each essay code. For each regression equation, in Step 1, the psychological or physical well-being score at T1, objective threat, word count, gender, age, and number of positive and negative emotion words were included as covariates. In Step 2, ethnicity and essay codes were entered. In the final step, the interactions between ethnicity and essay codes were entered.

\section{Results}

\section{Preliminary Analyses}

Preliminary analyses indicated that the cultural groups were matched on gender, $\chi^{2}=1.07, n s$; age, $t(32)=0.93, n s$; number of positive emotion words, $t(32)=0.88, n s$; number of negative emotion words, $t(32)=0.65, n s$; and objective threat of the events, $t(32)=0.71, n s$. We examined correlations between essay codes with T1 and T2 psychological and physical functioning outcomes across ethnic groups (see Table 2 for descriptive statistics and Table 3 for zero-order correlations). Contrary to predictions, independent samples $t$ tests showed that cultural groups did not differ significantly in the frequency of self-relevant motivations $(p s>.05)$. We also examined frequency of essay codes using 
Table 2

Means (and Standard Deviations) Between Asian Americans and European Americans on Essay Codes and Psychological and Physical Well-Being

\begin{tabular}{|c|c|c|c|c|c|}
\hline Variable & \multicolumn{2}{|c|}{ Asian Americans $(n=17)$} & \multicolumn{2}{|c|}{ European Americans $(n=17)$} & $p$ \\
\hline \multicolumn{6}{|l|}{ Essay codes ${ }^{\mathrm{a}}$} \\
\hline Situational attribution & \multicolumn{2}{|c|}{$4.18(2.83)$} & \multicolumn{2}{|c|}{$4.76(5.74)$} & $n s$ \\
\hline Downward social comparison & \multicolumn{2}{|c|}{$1.00(1.22)$} & \multicolumn{2}{|c|}{$0.65(1.00)$} & ns \\
\hline Upward social comparison & \multicolumn{2}{|c|}{$0.47(0.80)$} & \multicolumn{2}{|c|}{$0.53(1.18)$} & $n s$ \\
\hline Persistence & \multicolumn{2}{|c|}{$2.76(2.11)$} & \multicolumn{2}{|c|}{$0.94(1.60)$} & $n s$ \\
\hline \multicolumn{6}{|l|}{ Covariates } \\
\hline Positive emotion words & \multicolumn{2}{|c|}{$12.62(3.50)$} & \multicolumn{2}{|c|}{$11.57(3.49)$} & $n s$ \\
\hline Negative emotion words & \multicolumn{2}{|c|}{$12.71(3.44)$} & \multicolumn{2}{|c|}{$13.61(4.49)$} & $n s$ \\
\hline Age & \multicolumn{2}{|c|}{$20.24(1.15)$} & \multicolumn{2}{|c|}{$21.29(4.55)$} & $n s$ \\
\hline \multirow[t]{2}{*}{ Objective threat of the event } & \multicolumn{2}{|c|}{$2.63(0.67)$} & \multicolumn{2}{|c|}{$2.79(0.61)$} & $n s$ \\
\hline & Time 1 & Time 2 & Time 1 & Time 2 & \\
\hline Depressive symptoms & $-0.33(0.52)$ & $0.03(1.00)$ & $-0.07(0.79)$ & $0.29(1.04)$ & $n s$ \\
\hline Anxious symptoms & $-0.07(0.70)$ & $-0.29(0.52)$ & $-0.01(0.62)$ & $0.46(1.29)$ & $n s$ \\
\hline Physical symptoms & $98.06(20.22)$ & $102.24(25.39)$ & $108.12(30.03)$ & $107.06(24.13)$ & $n s$ \\
\hline
\end{tabular}

Note. Independent samples $t$ tests showed that all study variables were not significantly different between ethnic groups.

${ }^{a}$ Means and standard deviations of essay codes are the number of line blocks (i.e., 5 lines/block).

multilevel mixed-effects linear regression by the ordinal position of the essay (i.e., first through fourth essays), and found no significant change across time $\left(\chi^{2}\right.$ range $\left.=0.30-2.32, n s\right)$. To compare the degree of relatedness among essay codes, we assessed the number of line blocks double-coded. Essay codes shared a small degree of overlap: $2 \%$ in upward social comparison and persistence, $3 \%$ in upward social comparison and situational attribution, $2 \%$ in persistence and downward social comparison, and $7 \%$ in persistence and situational attribution overlapped. There was no triple coding. Correlations between essay codes and frequency of positive and negative emotion words were not significant.

Table 3 displays cultural differences in the relationships between essay codes and EW outcomes. Downward social comparison writing was associated with poorer outcomes at both time points for Asian Americans ( $r$ range $=.60-.66, p<.05$ ) but not for European Americans ( $r$ range $=-.40$ to $-.09, n s$ ), and persistence writing was associated with poorer outcomes at $\mathrm{T} 2$ for European Americans $(r=.46, p<.05)$ but not for Asian Americans $(r$ range $=-.38$ to $-.41, n s)$. The differences between these correlation coefficients between ethnic groups (i.e., Asian Americans and European Americans) were significantly different from each other using Fisher $r$-to- $z$ transformations $(p s<.05)$.

\section{Self-Enhancement: Downward Social Comparison and Situational Attributions}

Regression analyses revealed significant interactions of Ethnicity $\times$ Downward Social Comparison Writing on anxiety, $F(1$, 21) $=5.30, p<.05, \Delta R^{2}=12.67 \%, \eta_{\mathrm{p}}^{2}=.20$, and depressive symptoms, $F(1,21)=3.50, p<.05, \Delta R^{2}=8.99 \%, \eta_{\mathrm{p}}^{2}=.14$, but not on physical symptoms (see Figure 1). Simple slopes plotted in Figure 1 revealed that downward social comparison writing was positively associated significantly with an increase in anxiety and depressive symptoms for Asian Americans $(B=0.27$ and 0.37 , $p<.05$, respectively). In contrast, downward comparison predicted a significant improvement in anxiety for European Ameri- cans $(B=-0.30, p<.05$; depressive symptoms in the same direction, although not statistically significant).

We found a significant interaction of Ethnicity $\times$ Situational Attribution on depressive symptoms, $F(1,21)=7.85, p<.01$, $\Delta R^{2}=17.68 \%, \eta_{\mathrm{p}}^{2}=.27$, and a marginally significant interaction of Ethnicity $\times$ Situational Attribution on anxiety symptoms, $F(1$, $21)=3.64, p=.07, \Delta R^{2}=8.83 \%, \eta_{\mathrm{p}}^{2}=.15$. Simple slopes plotted in Figure 2 reveal that situational attribution was significantly associated with an increase in anxiety and depressive symptoms at $\mathrm{T} 2$ for Asian Americans $(B=0.22$ and $0.28, p<.05$, respectively), but approached zero and was not significant.

\section{Self-Improvement: Upward Social Comparison and Persistence}

Regression analyses revealed no significant interactions of Ethnicity $\times$ Upward Social Comparison Writing in predicting psychological and physical well-being. There were significant interactions of Ethnicity $\times$ Persistence Writing on depressive symptoms, $F(1,21)=$ $10.38, p<.01, \Delta R^{2}=21.13 \%, \eta_{\mathrm{p}}^{2}=.33$, and anxiety symptoms, $F(1$, $21)=2.31, p<.05, \Delta R^{2}=5.24 \%, \eta_{\mathrm{p}}^{2}=.10$, but not on physical symptoms. Simple slopes plotted in Figure 3 reveal that persistence writing was associated with an increase in anxiety and depressive symptoms at T2 for European Americans $(B=0.39$ and $0.30, p<$ .05 , respectively), but was associated with a significant improvement in depressive, but not anxiety symptoms for Asian Americans $\left(B=-1.14\right.$ and $-0.26, p<.01$ and $n s$, respectively) ${ }^{1}$

\footnotetext{
${ }^{1}$ The inclusion of the set of covariates did not substantially influence the significance or direction of effects in any of the reported results with the exception of situational attributions in predicting depressive symptoms when the set of covariates was not included in the model (i.e., from $B=$ $0.28, p<.05$, to $B=0.16, n s)$.
} 

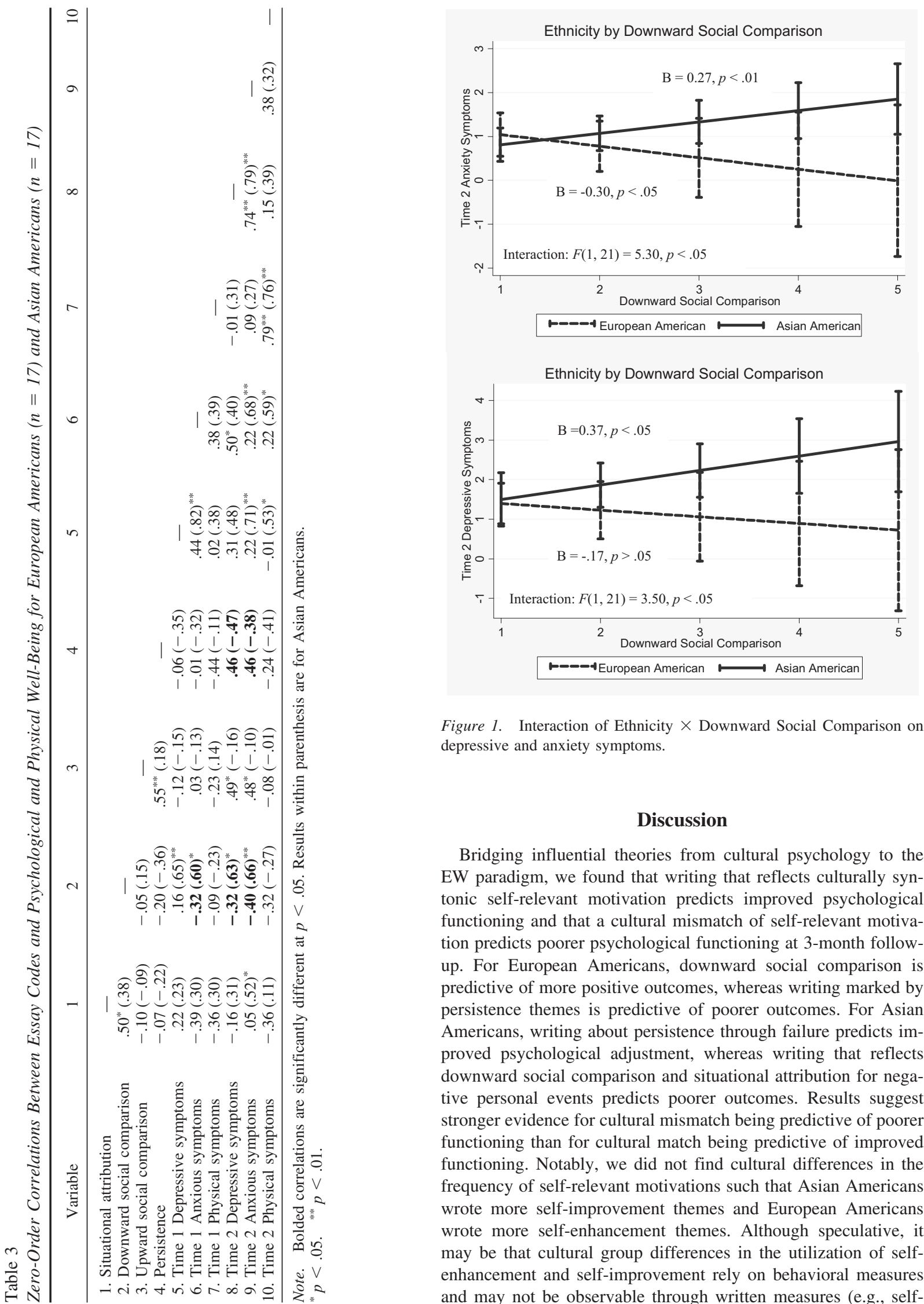

Figure 1. Interaction of Ethnicity $\times$ Downward Social Comparison on depressive and anxiety symptoms.

\section{Discussion}

Bridging influential theories from cultural psychology to the EW paradigm, we found that writing that reflects culturally syntonic self-relevant motivation predicts improved psychological functioning and that a cultural mismatch of self-relevant motivation predicts poorer psychological functioning at 3-month followup. For European Americans, downward social comparison is predictive of more positive outcomes, whereas writing marked by persistence themes is predictive of poorer outcomes. For Asian Americans, writing about persistence through failure predicts improved psychological adjustment, whereas writing that reflects downward social comparison and situational attribution for negative personal events predicts poorer outcomes. Results suggest stronger evidence for cultural mismatch being predictive of poorer functioning than for cultural match being predictive of improved functioning. Notably, we did not find cultural differences in the frequency of self-relevant motivations such that Asian Americans wrote more self-improvement themes and European Americans wrote more self-enhancement themes. Although speculative, it may be that cultural group differences in the utilization of selfenhancement and self-improvement rely on behavioral measures and may not be observable through written measures (e.g., self- 


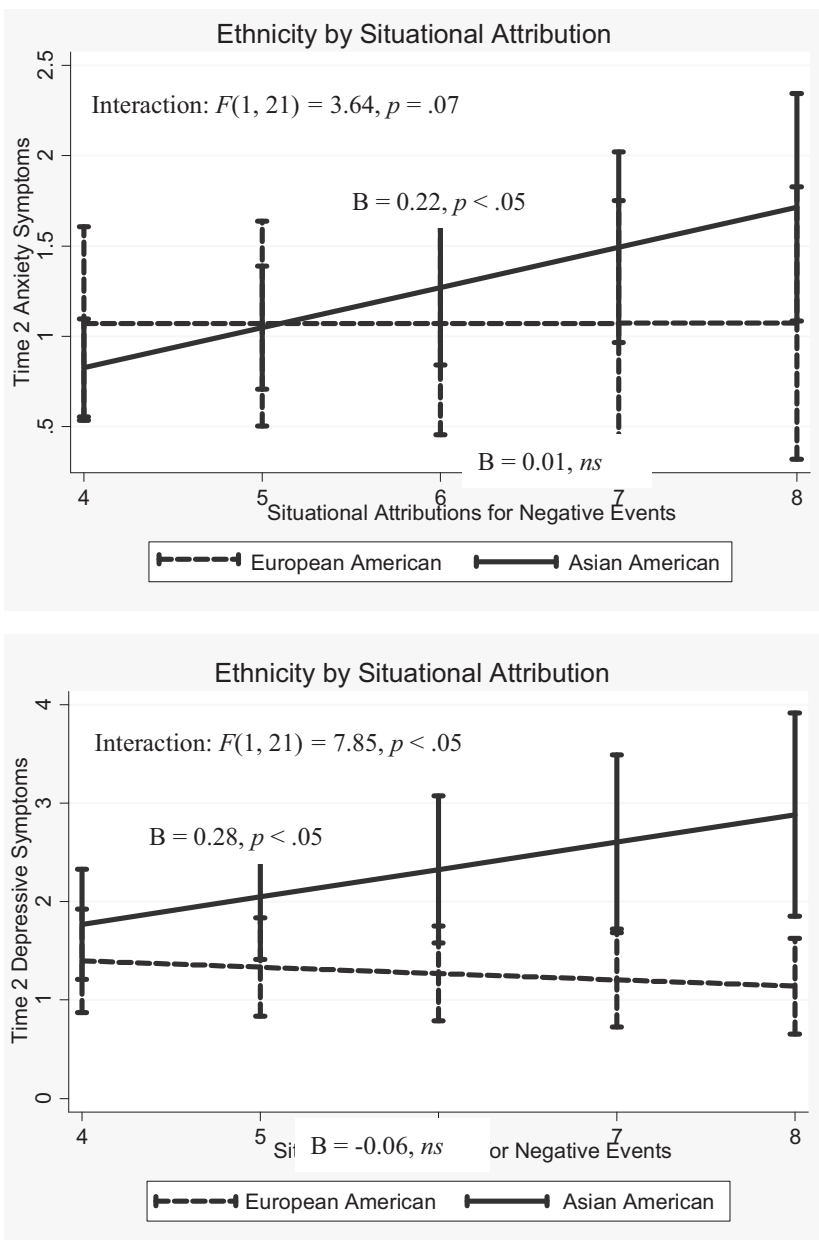

Figure 2. Interaction of Ethnicity $\times$ Situational Attribution on depressive and anxiety symptoms.

report; Heine, Lehman, Peng, \& Greenholtz, 2002). Indeed, the majority of studies have demonstrated cultural differences through examining behavioral measures of self-relevant motivations (e.g., time persisting on task; Heine \& Raineri, 2009).

Writing about the importance of persisting through failure appears to confer important emotional benefits for Asian Americans. Previous experimental findings have revealed a greater tendency on the part of Easterners to focus on areas of relative weakness, arguably to allow for the opportunity to self-correct. However, the current findings are novel, suggesting that when individuals of Asian descent focus on persisting in areas of difficulty, they reap psychological benefits as well as potential performance advantages. On the other hand, making downward social comparisons and attributing failure outcomes to external circumstances may reflect attempts to deflect poor outcomes or negative evaluations. One possibility is that among cultural groups for whom selfenhancement is less normative, downward social comparison in the context of negative personal experiences may more likely be a reflection of trait negative affectivity (e.g., resentment), which may not palliate distress. Indeed, inconsistency with selfimproving orientation in EW appeared to undermine benefits for Asian Americans.
In contrast, downward social comparison was beneficial for European Americans. This may be taken as indirect evidence that European Americans subscribe to entity theories of the self as relatively immutable, resulting in negative information about the self becoming relatively more threatening. Downward social comparison may help European Americans focus on areas of strength to maintain positive self-regard. Although our results show situational attributions for negative personal events to be unrelated to EW outcomes for European Americans, the positive relationship between downward social comparison and situational attributions provides support for the notion that both processes subserve selfenhancement motives. This dovetails with findings from Creswell and colleagues (2007) that self-affirmation (i.e., writing about valued aspects of the self) predicted better outcomes in their primarily European American sample. It is plausible that for European Americans, tenacious persistence in areas of previous failure may present some risk to emotional well-being, as this orientation may continue to expose individuals to threats to the self. Effort to restore self-efficacy by diverting attention from negative self-relevant information is congruent with a self-enhancement motivation.

Our findings may also inform efforts to reconcile previous contradictory findings about the benefits of EW across cultural groups. Lu and Stanton (2010) found that Asian Americans ben-

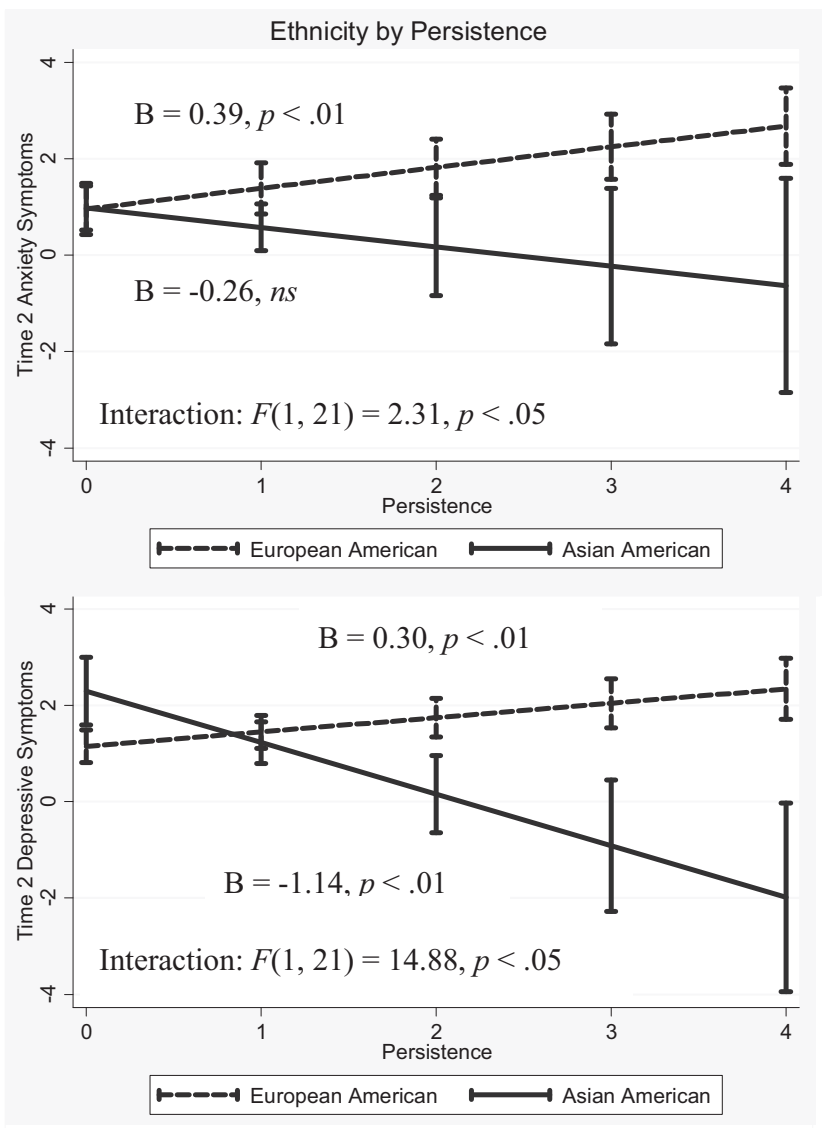

Figure 3. Interaction of Ethnicity $\times$ Persistence on depressive and anxiety symptoms. 
efitted more from EW compared with European Americans, whereas Knowles et al. (2011) reported the opposite pattern. Whereas Lu and Stanton instructed writers to use cognitive reappraisal, Knowles et al. instructed participants simply to write about their deepest thoughts and feelings. Perhaps the cognitive reappraisal instructional set shaped a more culturally congruent selfimprovement focus among Asian Americans in the Lu and Stanton study.

Self-enhancement and self-improvement motivations were not differentially related to physical symptom outcomes across the two cultural groups. This was unexpected given that stronger intervention effects have been noted for physical symptoms than for psychological symptoms (Frattaroli, 2004). Perhaps psychological symptoms are more closely related to the EW processes revealed in self-relevant motivations than physical symptoms. It is also possible that cultural differences in the mechanisms of EW may be more easily detected in affective outcomes.

Although this study provides compelling data on cultural variability in the function of self-enhancement and self-improvement motivations in predicting psychological well-being in EW, it also has several limitations. First, the present study had a small sample revealing large effect sizes, but future replication is essential as small sample sizes could lead to inaccurate and spurious findings (Schönbrodt \& Perugini, 2013). Second, it was not possible to examine within-group variability (e.g., generational status or Asian subgroups); results may not generalize to the larger Asian American and European American community. Third, we did not manipulate self-relevant motivations directly. Instead, we assessed the naturalistic occurrence of these motives through writing. Although this limitation restricts our ability to make causal statements, we had the opportunity to compare the naturalistic use of these foci with tests underlying psychological mechanisms in EW (also see Niles et al., in press, in preparation). Lastly, we used ethnic group membership as a proxy for cultural differences in self-construals. Future research should directly assess independent and interdependent self-construals to provide more direct evidence of the culturally based theories posed.

Despite these limitations, this study used a novel approach for assessing potential psychological mechanisms of expressive disclosure and provided empirical evidence supporting the links between self-relevant motivations and outcomes. In EW, we found that self-enhancement motivation in the form of downward social comparison is adaptive for European Americans, but not for Asian Americans. In contrast, self-improvement motivation in the form of persistence themes is adaptive for Asian Americans, but not for European Americans. Findings from the present research suggest the importance of future work focused on the roles of cultural values and processes to clarify our understanding of the mechanisms underlying the ameliorative effects of EW.

\section{References}

Antony, M. M., Bieling, P. J., Cox, B. J., Enns, M. W., \& Swinson, R. P. (1998). Psychometric properties of the 42-item and 21-item versions of the Depression Anxiety Stress Scales in clinical groups and a community sample. Psychological Assessment, 10, 176-181. doi:10.1037/10403590.10.2.176

Bandura, A. (1999). Social cognitive theory of personality. In D. Cervone $\&$ Y. Shoda (Eds.), The coherence of personality: Social cognitive bases of personality consistency, variability, and organization (pp. 185-241). New York, NY: Guilford Press.

Beck, A. T., \& Steer, R. A. (1984). Internal consistencies of the original and revised Beck Depression Inventory. Journal of Clinical Psychology, 40, 1365-1367. doi:10.1002/1097-4679(198411)40:6<1365::AIDJCLP2270400615>3.0.CO;2-D

Butler, R. (1992). What young people want to know when: Effects of mastery and ability goals on interest in different kinds of social comparisons. Journal of Personality and Social Psychology, 62, 934-943. doi:10.1037/0022-3514.62.6.934

Chung, T., \& Mallery, P. (1999). Social comparison, individualismcollectivism, and self-esteem in China and the United States. Current Psychology, 18, 340-352. doi:10.1007/s12144-999-1008-0

Creswell, J. D., Lam, S., Stanton, A. L., Taylor, S. E., Bower, J. E., \& Sherman, D. K. (2007). Does self-affirmation, cognitive processing, or discovery of meaning explain cancer-related health benefits of expressive writing? Personality and Social Psychology Bulletin, 33, 238-250. doi: $10.1177 / 0146167206294412$

Crocker, J., Thompson, L. L., McGraw, K. M., \& Ingerman, C. (1987). Downward comparison prejudice and evaluations of others: Effects of self-esteem and threat. Journal of Personality and Social Psychology, 52, 907-916. doi:10.1037/0022-3514.52.5.907

Derogatis, L. R., \& Melisaratos, N. (1983). The Brief Symptom Inventory: An introductory report. Psychological Medicine, 13, 595-605. doi: 10.1017/S0033291700048017

Diener, E. (1984). Subjective well-being. Psychological Bulletin, 95, 542 575. doi:10.1037/0033-2909.95.3.542

Francis, M. E., \& Pennebaker, J. W. (1993). LIWC: Linguistic Inquiry and Word Count. Dallas, TX: Southern Methodist University.

Frattaroli, J. (2006). Experimental disclosure and its moderators: A metaanalysis. Psychological Bulletin, 132, 823-865. doi:10.1037/0033-2909 .132 .6 .823

Guttman, I. (1973). Care and handling of univariate or multivariate outliers in detecting spuriosity: A Bayesian approach. Technometrics, 20, 723 738 .

Hammen, C., Marks, T., Mayol, A., \& DeMayo, R. (1985). Depressive self-schemas, life stress, and vulnerability to depression. Journal of Abnormal Psychology, 94, 308-319. doi:10.1037/0021-843X.94.3.308

Heine, S. J., \& Hamamura, T. (2007). In search of East Asian selfenhancement. Personality and Social Psychology Review, 11, 4-27. doi:10.1177/1088868306294587

Heine, S. J., Kitayama, S., Lehman, D. R., Takata, T., Ide, E., Leung, C., \& Matsumoto, H. (2001). Divergent consequences of success and failure in Japan and North America: An investigation of self-improving motivations and malleable selves. Journal of Personality and Social Psychology, 81, 599-615. doi:10.1037/0022-3514.81.4.599

Heine, S. J., Lehman, D. R., Markus, H. R., \& Kitayama, S. (1999). Is there a universal need for positive self-regard? Psychological Review, 106, 766-794. doi:10.1037/0033-295X.106.4.766

Heine, S. J., Lehman, D. R., Peng, K., \& Greenholtz, J. (2002). What's wrong with cross-cultural comparisons of subjective Likert scales?: The reference-group effect. Journal of personality and social psychology, 82, 903-918.

Heine, S. J., \& Raineri, A. (2009). Self-improving motivations and collectivism the case of Chileans. Journal of Cross-Cultural Psychology, 40, $158-163$.

Kang, S., Shaver, P. R., Sue, S., Min, K., \& Jing, H. (2003). Culturespecific patterns in the prediction of life satisfaction: Roles of emotion, relationship quality, and self-esteem. Personality and Social Psychology Bulletin, 29, 1596-1608. doi:10.1177/0146167203255986

Kitayama, S., Markus, H. R., Matsumoto, H., \& Norasakkunkit, V. (1997). Individual and collective processes in the construction of the self: Self-enhancement in the United States and self-criticism in Japan. Jour- 
nal of Personality and Social Psychology, 72, 1245-1267. doi:10.1037/ 0022-3514.72.6.1245

Knowles, E. D., Wearing, J. R., \& Campos, B. (2011). Culture and the health benefits of expressive writing. Social Psychological and Personality Science, 2, 408-415. doi:10.1177/1948550610395780

Lepore, S. J., \& Greenberg, M. A. (2002). Mending broken hearts: Effects of expressive writing on mood cognitive processing, social adjustment, and health following a relationship breakup. Psychology \& Health, 17, 547-560. doi:10.1080/08870440290025768

Lu, Q., \& Stanton, A. L. (2010). How benefits of expressive writing vary as a function of writing instructions, ethnicity and ambivalence over emotional expression. Psychology \& Health, 25, 669-684. doi:10.1080/ 08870440902883196

Markus, H. R., \& Kitayama, S. (1991). Culture and the self: Implications for cognition, emotion, and motivation. Psychological Review, 98, 224253. doi: $10.1037 / 0033-295 X .98 .2 .224$

Markus, H. R., \& Kitayama, S. (2010). Cultures and selves: A cycle of mutual constitution. Perspectives on Psychological Science, 5, 420430. doi:10.1177/1745691610375557

Mezulis, A. H., Abramson, L. Y., Hyde, J. S., \& Hankin, B. L. (2004). Is there a universal positivity bias in attributions? A meta-analytic review of individual, developmental, and cultural differences in the self-serving attributional bias. Psychological Bulletin, 130, 711-747. doi:10.1037/ 0033-2909.130.5.711

Niles, A. N., Haltom, K. B., Hur, C., Lieberman, M. D., \& Stanton, A. L. (2014). Testing discovery of meaning, self affirmation, exposure, and narrative structure as mechanisms of the benefits of expressive writing. Manuscript submitted for publication.

Niles, A. N., Haltom, K. E., Mulvenna, C. M., Lieberman, M. D., \& Stanton, A. L. (in press). Effects of expressive writing on psychological and physical health: The moderating role of emotional expressivity. Anxiety, Stress \& Coping.

Pennebaker, J. W. (1982). The psychology of physical symptoms. New York, NY: Springer-Verlag. doi:10.1007/978-1-4613-8196-9

Pennebaker, J. W., Mayne, T. J., \& Francis, M. E. (1997). Linguistic predictors of adaptive bereavement. Journal of Personality and Social Psychology, 72, 863-871. doi:10.1037/0022-3514.72.4.863
Radloff, L. S. (1977). The CES-D scale: A self-report depression scale for research in the general population. Applied Psychological Measurement, 1, 385-401. doi:10.1177/014662167700100306

Ross, M., Heine, S. J., Wilson, A. E., \& Sugimori, S. (2005). Cross-cultural discrepancies in self-appraisals. Personality and Social Psychology Bulletin, 31, 1175-1188. doi:10.1177/0146167204274080

Schönbrodt, F. D., \& Perugini, M. (2013). At what sample size do correlations stabilize? Journal of Research in Personality, 47, 609-612. doi:10.1016/j.jrp.2013.05.009

Smyth, J. M. (1998). Written emotional expression: Effect sizes, outcome types, and moderating variables. Journal of Consulting and Clinical Psychology, 66, 174-184. doi:10.1037/0022-006X.66.1.174

Steer, R. A., Beck, A. T., Garrison, B., \& Lester, D. (1988). Eventual suicide in interrupted and uninterrupted attempters: A challenge to the cry-for-help hypothesis. Suicide and Life-Threatening Behavior, 18, 119-128.

Stevenson, H. (1994). Learning gap: Why our schools are failing and what we can learn from Japanese and Chinese education. New York, NY: Simon \& Schuster.

Taylor, S. E., \& Brown, J. D. (1988). Illusion and well-being: A social psychological perspective on mental health. Psychological Bulletin, 103 , 193-210. doi:10.1037/0033-2909.103.2.193

Tesser, A., Campbell, J. D., \& Smith, M. (1984). Friendship choice and performance: Self-evaluation maintenance in children. Journal of Personality and Social Psychology, 46, 561-574. doi:10.1037/0022-3514 .46.3.561

White, K., \& Lehman, D. R. (2005a). Culture and social comparison seeking: The role of self-motives. Personality and Social Psychology Bulletin, 31, 232-242. doi:10.1177/0146167204271326

White, K., \& Lehman, D. R. (2005b). Looking on the bright side: Downward counterfactual thinking in response to negative life events. Personality and Social Psychology Bulletin, 31, 1413-1424. doi:10.1177/ 0146167205276064

Wills, T. A. (1981). Downward comparison principles in social psychology. Psychological Bulletin, 90, 245-271. doi:10.1037/0033-2909.90.2 .245

\section{E-Mail Notification of Your Latest Issue Online!}

Would you like to know when the next issue of your favorite APA journal will be available online? This service is now available to you. Sign up at http://notify.apa.org/ and you will be notified by e-mail when issues of interest to you become available! 